\title{
Analysis of Frequency of Use of Different Scar Assessment Scales Based on the Scar Condition and Treatment Method
}

\author{
Seong Hwan Bae, Yong Chan Bae \\ Department of Plastic and Reconstructive Surgery, Pusan National University School of Medicine, Busan, Korea
}

\begin{abstract}
Analysis of scars in various conditions is essential, but no consensus had been reached on the scar assessment scale to select for a given condition. We reviewed papers to determine the scar assessment scale selected depending on the scar condition and treatment method. We searched PubMed for articles published since 2000 with the contents of the scar evaluation using a scar assessment scale with a Journal Citation Report impact factor $>0.5$. Among them, 96 articles that conducted a scar evaluation using a scar assessment scale were reviewed and analyzed. The scar assessment scales were identified and organized by various criteria. Among the types of scar assessment scales, the Patient and Observer Scar Assessment Scale (POSAS) was found to be the most frequently used scale. As for the assessment of newly developed operative scars, the POSAS was most used. Meanwhile, for categories depending on the treatment methods for preexisting scars, the Vancouver Scar Scale (VSS) was used in 6 studies following a laser treatment, the POSAS was used in 7 studies following surgical treatment, and the POSAS was used in 7 studies following a conservative treatment. Within the 12 categories of scar status, the VSS showed the highest frequency in 6 categories and the POSAS showed the highest frequency in the other 6 categories. According to our reviews, the POSAS and VSS are the most frequently used scar assessment scales. In the future, an optimal, universal scar scoring system is needed in order to better evaluate and treat pathologic scarring.
\end{abstract}

Keywords Cicatrix / Keloid / Visual analog scale
Correspondence: Yong Chan Bae Department of Plastic and Reconstructive Surgery, Pusan National University School of Medicine, 179 Gudeok-ro, Seo-gu, Busan 602-739, Korea

Tel: +82-51-240-7273

Fax: +82-51-243-9405

E-mail: baeyc2@hanmail.net

This work was supported by a 2-year research grant from Pusan National University.

This article was presented at the $3 \mathrm{rd}$ Research \& Reconstructive forum on May 9-10, 2013 in Daegu, Korea.

No potential conflict of interest relevant to this article was reported.

Received: 22 Apr $2013 \bullet$ Revised: 22 Jul $2013 \bullet$ Accepted: 23 Jul 2013

pISSN: 2234-6163 • elSSN: 2234-6171 • http://dx.doi.org/10.5999/aps.2014.41.2.111 • Arch Plast Surg 2014;41:111-115

\section{INTRODUCTION}

Scarring comprises various processes involving many factors, and scars have distinctive differences from normal tissues in terms of appearance and pathology. Scars have been classified on the basis of differences from normal tissues. However, currently, there is no definite established method for the classification of scars, and various criteria such as reasons for scar development and characteristics of the scars are concurrently used
[1].

Initially, a scar assessment scale for evaluating scars quantified scar appearances according to the treatment response, and this scale was continuously modified to objectively include subjective factors. The universally used scale was the Vancouver Scar Scale (VSS), which was developed by Sullivan et al. [2] in 1990 and calculates and aggregates points in 4 categories: the vascularity, pigmentation, pliability, and height of scars. However, the pigmentation category had a limited range of evaluation and it is

Copyright $($ C 2014 The Korean Society of Plastic and Reconstructive Surgeons

This is an Open Access article distributed under the terms of the Creative Commons Attribution Non-Commercial License (http://creativecommons.org/

licenses/by-nc/3.0/) which permits unrestricted non-commercial use, distribution, and reproduction in any medium, provided the original work is properly cited.

www.e-aps.org 
difficult to distinguish it from vascularity [1]. To resolve this, Beausang et al. [3] developed the Manchester Scar Scale (MSS) in 1998, which allows for concrete notation of visibleaspects of scars. Since the publication of the Patient and Observer Scar Assessment Scale (POSAS), there have also been attempts to include subjective symptoms such as pain and urtication, which had not been considered in previous scar assessment scales [4].

In 2011, Fearmonti et al. [5] defined the term "pathologic scarring" as not only an experience of symptoms of pain, urtication, and functional impairment but also an experience of the symptoms by which patients are inconvenienced in their daily life and for which they decide to pursue treatment. From this trend, we note that the concept of scarring has expanded in different directions from the initial view considering scarring to be a simple visual defect. Accordingly, scar assessment scales have been modified into various types as their scope of application has broadened. Unfortunately, with the diversification of the scar assessment scale, it has become more difficult to select and apply the most suitable scale; thus multiple scar assessment scales are being used in confusion.

Therefore, the scar status should be categorized on the basis of clinical conditions and treatment methods, and it is imperative that the appropriate scar assessment scale is selected and applied for a given purpose.

Consequently, we reviewed published articles to investigate and summarize the types of scar assessment scales used based on scar conditions and treatment methods.

\section{METHODS}

We searched PubMed for all articles published since 2000 with the contents of scar evaluation using a scar assessment scale. One hundred four articles met these criteria. To enhance the credibility of this study, articles with a Journal Citation Report impact factor lower than 0.5 were excluded. Studies that used 2 or more scar assessment scales concurrently were excluded. However, in studies that used concurrent multiple scar assessment scales, the scale that was considered better was included in our study if there was a clear conclusion on the comparative statistical analysis as well as its preference. Finally, cases with the concurrent use of 2 or more treatment methods (e.g., the use of both surgical and laser treatments) were excluded from the study.

Finally, 96 articles were reviewed for study inclusion. The cause of scar development, clinical condition, location, and treatment methods were analyzed from all of the articles, and the scar assessment scale used in each situation was categorized thereafter.
First, all of the scar assessment scales used were categorized, and the selection frequency was identified for each. Five scar assessment scales were used frequently: the VSS, POSAS, Visual Analog Scale (VAS), MSS, and the Stony Brook Scar Evaluation Scale (SBSES). Other scar assessment scales were classified into an "other" group because of relatively low frequency and a lack of objectivity.

Next, the cases in which a scar assessment scale was used were analyzed to evaluate the results of certain procedures. Specifically, we considered the types of scar assessment scales that were used to assess newly developed operative scars. In addition, we classified the use of scar assessment scales for different treatment methods in the presence of preexisting scars by selection frequency. The treatment methods used for the scars can be classified into 3 categories: laser treatment, surgical treatment, and conservative treatment. Among them, the conservative treatment comprised non-invasive treatments, excluding laser treatment, such as massage and silicone sheet application.

Lastly, the overall scar status was separated into categories on the basis of cause of development, clinical manifestation, location, treatment method, and more. Furthermore, different scar assessment scales were identified for each case. The scars were largely classified into operative scars and preexisting scars. The newly developed operative scars were then classified into linear scars and widespread scars, in which linear scars were categorized separately as limited to the head and neck region and various other regions.

The scars caused by burns, injuries, operations, and other means that were treated with a laser or surgery were defined as preexisting scars. Preexisting scars were classified according to the cause and clinical manifestation, and they were divided into 3 categories of burn/traumatic scars (non-keloid/hypertrophic scars) postoperative scars (non-keloid/hypertrophic scars) and keloid/hypertrophic scars. As mentioned earlier, the treatment methods for each scar were classified into 3 categories of laser, surgical, and conservative treatment. The scar assessment scales used in each case were noted to be VSS, POSAS, VAS, MSS, or SBSES; other scales were classified as "other."

\section{RESULTS}

Among the scar assessment scales, the POSAS was the most frequently used scale, used in 35 studies (37.5\%), followed by the VSS, which was used in 33 studies (34.4\%) (Table 1). In all, 54 studies had used scar assessment scales for the outcome assessment of newly developed operative scars after certain procedures. Overall, the POSAS was most used for operative scar assessment (19 studies, 35.2\%) followed by the VSS (17 stud- 
ies, 31.5\%). For categories depending on treatment methods for preexisting scars, the VVS was most used after laser treatment (6 studies, $54.5 \%$ ), the POSAS was most used after surgery (7 studies, $53.8 \%$ ), and the POSAS was also most used after conservative treatment ( 7 studies, $38.9 \%$ ) followed by the VSS (6 studies, 33.3\%) (Table 2).

Among all of the 12 categories based on the cause, clinical manifestation, location, and treatment methods of scar status, the VSS had the highest frequency in 6 categories, and the POSAS had the highest frequency in the other 6 categories. Among them, in 1 category, the POSAS and VSS both showed the highest frequency. In another category, the POSAS and the VAS showed the highest frequency (Fig. 1).

\section{DISCUSSION}

Numerous scars caused by surgery, injuries, and burns are report-

\section{Table 1. The application rate of each scar assessment scale}

\begin{tabular}{|lcc|}
\hline Scar assessment scale & No. of articles & Proportion (\%) \\
\hline POSAS & 35 & 37.5 \\
VSS & 33 & 34.4 \\
VAS & 17 & 17.7 \\
MSS & 3 & 3.1 \\
SBSES & 1 & 1.0 \\
Other & 7 & 7.3 \\
Total & 96 & \\
\hline POSAS, Patient and Observer Scar Assessment Scale; VSS, Vancouver Scar Scale; \\
VAS, Visual Analog Scale; MSS, Manchester Scar Scale; SBSES, Stony Brook Scar \\
Evaluation Scale.
\end{tabular}

ed annually, and they are classified depending on their cause, depth, and area. It is important to assess scars according to their relevant characteristics and context. First, this should provide an objective criterion for treatment outcomes. Second, it would aid in the establishment of appropriate treatment outcomes. In this regard, a scar assessment scale can be deemed as meaningful and will influence doctors, patients, and researchers [6].

The VVS, which is universally used for assessing scars caused by burns, was first published by Sullivan et al. [2] in 1990. The VSS assesses and calculates subscores in 4 categories (i.e., vascularity, pigmentation, pliability, and height of the scar) and aggregates the scores [1]. The pigmentation category is simplified into 3 states: normal, hypopigmentation, and hyperpigmentation. This assessment method lacks clarity in a clinical setting,

Table 2. The application of scar assessment scales used to evaluate the results of procedures

\begin{tabular}{|c|c|c|c|}
\hline Type of scar & $\begin{array}{l}\text { Treatment } \\
\text { method }\end{array}$ & $\begin{array}{l}\text { No. of } \\
\text { articles }\end{array}$ & $\begin{array}{l}\text { Scar assessment scale } \\
\text { (no. of articles) }\end{array}$ \\
\hline Operative scar & - & 54 & $\begin{array}{l}\text { POSAS (19), VSS (17), VAS (10), } \\
\text { others (5), MSS (3) }\end{array}$ \\
\hline \multirow[t]{3}{*}{ Preexisting scar } & Laser treatment & 11 & $\begin{array}{l}\text { VSS (6), POSAS (2), others (2), } \\
\text { VAS (1) }\end{array}$ \\
\hline & Surgical treatment & 13 & $\begin{array}{l}\text { POSAS (7), VSS (4), VAS (1), } \\
\text { SBSES (1) }\end{array}$ \\
\hline & $\begin{array}{l}\text { Conservative } \\
\text { treatment }\end{array}$ & 18 & POSAS (7), VSS (6), VAS (5) \\
\hline Total & & 96 & \\
\hline \multicolumn{4}{|c|}{$\begin{array}{l}\text { POSAS, Patient and Observer Scar Assessment Scale; VSS, Vancouver Scar Scale; } \\
\text { VAS, Visual Analog Scale; MSS, Manchester Scar Scale; SBSES, Stony Brook Scar } \\
\text { Evaluation Scale. }\end{array}$} \\
\hline
\end{tabular}

\section{Fig. 1. Summary of the application of scar assessment scales according to the type of scars}

POSAS, Patient and Observer Scar Assessment Scale; VAS, Visual Analog Scale; VSS, Vancouver Scar Scale; MSS, Manchester Scar Scale; SBSES, Stony Brook Scar Evaluation Scale.

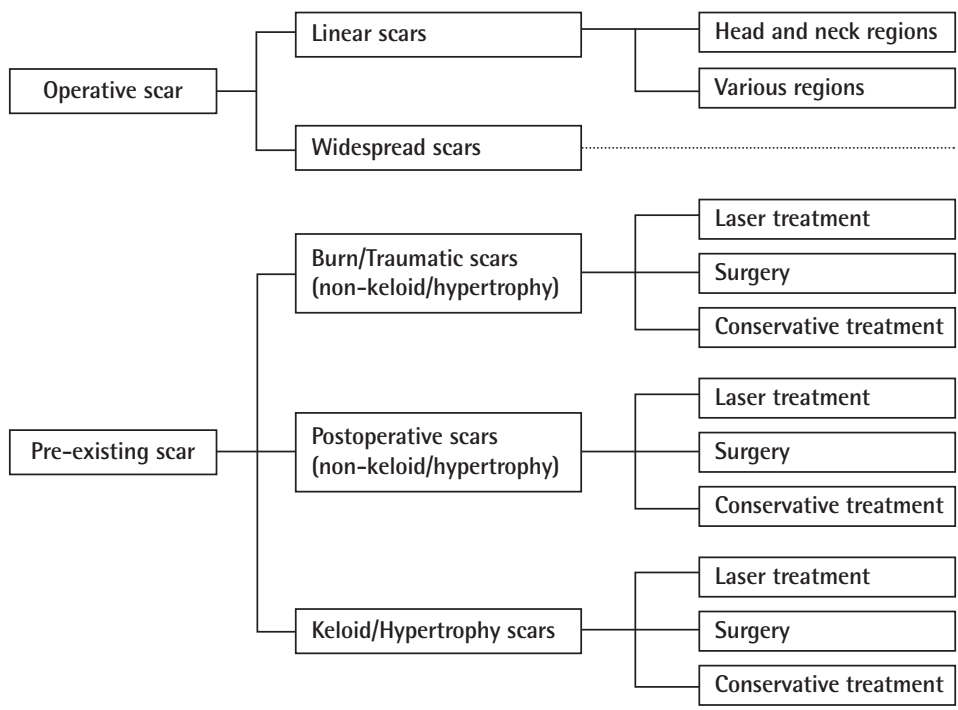

POSAS (5), VAS (5), VSS (3), others (3)

POSAS (11), VSS (9), VAS (4), MSS (3), others (2)

VSS (5), POSAS (3), VAS (1)

VSS (2), POSAS (1)

POSAS (3), VSS (3), VAS (1)

POSAS (3), VSS (2)

VSS (3), POSAS (1), VAS (1), others (1)

VSS (1)

VAS (4), VSS (3), POSAS (2)

VSS (1), others (1)

POSAS (4), SBSES (1)

POSAS (2), VAS (1), VSS (1) 
and in certain cases, the distinction between pigmentation and vascularity is vague. In addition, it does not include factors of functional mobility such as contracture into the pliability category $[1,6]$.

In 1995, Quinn et al. [7] assessed the cosmetic outcomes of scars after skin injury treatment using the VAS. They considered that conventional scales assess pain and cosmetic factors in a subjective manner; therefore, the scale needs to be able to objectively assess scars. The researchers performed assessments using the VAS by showing pictures of 33 scar cases to 4 cosmetic surgeons, and the objective assessments were made from a cosmetic perspective via interobserver agreement [8].

In 1998, Beausang et al. [3] presented the MSS, which is different from conventional scar assessment scales, in that it includes the mismatch level between the scars and the surrounding skin. Color, matte/shiny, contour, distortion, and texture were proposed as assessment criteria, and in particular, points were suggested by recording the level of mismatch with the surrounding skin in color and contour criteria. Compared with the conventional VSS, this allows clinicians to make a more detailed assessment of the scar appearance and is more clinically useful for the assessment of widespread scars among postoperative scars [6].

In 2004, Draaijers et al. [4] presented the POSAS, which comprises two factors: an observer scar assessment scale and a patient scar assessment scale. The method is significant because it includes subjective symptoms beyond the objective-oriented attempts and emphasizes the importance of the subjective symptoms of patients. Subjective symptoms were assessed by considering the criteria of pain, itching, color, stiffness, thickness, and relief, and in particular, it has been evaluated as the only scar assessment scale that includes pain and pruritus [9]. According to Truong et al. [8], the POSAS may be particularly useful for linear scar assessment after breast cancer operations, and when the observer scar assessment scale was compared with the VSS, it was statistically proven to have favorable outcomes in internal consistency and interobserver reliability.

The SBSES was publicized by Singer et al. [9] in 2007 and comprises 5 factors: width, height, color, suture marks, and overall appearance. Each factor is reclassified into 2 items, and 0 or 1 point is added before summing them up. It is appropriate for shortterm cosmetic outcomes of approximately 5 to 10 days after the stitches are removed, but along with functional scar evaluation, it is difficult to apply it to long-term cosmetic outcomes [6].

In 2011, Fearmonti et al. [5] defined "scar" with the term "pathologic scarring" to include symptoms of pain and urtication along with functional impairments that are severe enough that they inconvenience patients in their daily activities and functional abilities. They attempted to differentiate it from "non- pathologic scarring," which is generated in the process of the normal treatment of woundsand does not have specific symptoms, and also tried to include it in a range of disease.

Thus, the concept of scarring has been expanded from being limited to aesthetic aspects to encompassing functional and symptomatic aspects. In line with this development, there have been attempts to include a wide variety of items into scar assessment scales, and depending on the case, there have also been attempts to specifically describe certain parts. Because of the diversity of scar assessment scales, there have been difficulties selecting and applying the desired scale for the desired purposes.

Among the studies published recently, some have mentioned the statistical superiority of certain scar assessment scales for certain scar conditions or certain treatments, but no study has clarified the method for selecting a scar assessment scale that fits a given circumstance after classifying the scar status according to the treatment method or clinical condition. There has been a lack of research on the selection and application of a desirable scar assessment scale for each circumstance after appropriate classification when, in reality, the concept of scarring is expanding.

For these reasons, we reviewed the papers describing scar evaluation using a scar assessment scale. We summarized the various type of scars depending on the cause of development and clinical condition and reorganizde them according to the treatment method to evaluate which scar assessment scale is being used for assessing the status of a specific type of scar from a clinical perspective. Our reviews show that along with the VSS, which is deemed to be broadlyused, the POSAS has been used with the highest frequency; it was used in more than $70 \%$ of the studies. In particular, the fact that among scar assessment scales used in since 2000, the selection frequency of the POSAS including the patient component was higher than that of the VSS, which is a good indicator that the concept of scarring has expanded as mentioned earlier. Draaijers et al. [4] made attempts at statistical comparison between the observer component of POSAS and VSS, and based on the research outcome, the POSAS was more favorable in consistency and reliability; hence, they claimed that the POSAS was more suitable for scar assessmen. Additionally, in 2012, Nicholas et al. [10] claimed that the POSAS was more suitable for scar assessment based on research finding that the observer component of the POSAS was consistent and reliable for keloid scar assessment. Considering such circumstances, the evaluation of the VSS, which has been deemed to be widely usedfor scar assessment up to now, may change in the future. Considering the advantages of a broader application potential and statistically higher reliability in various conditions, the POSAS is considred to be used more widely than VSS. Our study showed that outcome assessments after certain procedures in 
which the scar assessment scale was used are important for providing information on the desirable scar assessment scale based on the treatment method for objective comparison. This research indicates that along with the POSAS, the VSS has been most frequently used, and in particular, the POSAS was most used in 3 categories out of 4 . It shows that unlike the past, wherein the treatment provider or the medical staff made objective decisions on the treatment outcome evaluation, currently, the focus has shifted to the subjective symptoms or opinions of patients. Considering this, the importance of patients' opinions can be further emphasized in the future assessment of treatment outcomes; therefore, it seems necessary to develop a scar assessment scale that accentuates these factors.

Analyzing scars on a scar assessment scale that considered each circumstance after classifying the scar status into 12 categories (based on the scar's cause of development, clinical manifestation, location, and treatment method) showed that the POSAS and VSS have been used widely. Nonetheless, the absolute number of scar assessment scales used in each type of circumstance was irregular, and Among 12 categories, several categories have only excessively low absolute numbers of case, inhibiting us from drawing a clear conclusion.

\section{CONCLUSIONS}

Generally, the POSAS and VSS have been widely used for scar assessment, but it was difficult to decide which scar assessment scale was used typically when each scar condition and treatment method has been considered.

Clinical scar assessment lacks a standardized methodology and a systematic approach, and thus studies continue to lack consensus regarding the most appropriate and applicable evaluation instrument. Refinement of scar assessment methods will serve to facilitate our treatment and prevention of scar formation. A comprehensive universal scar scoring system should be developed in order to assess various scar statuses and treat pathologic scarring.
The data obtained in this review has provided significant information on the latest trends in the selection of scar assessment scales.

\section{REFERENCES}

1. van Zuijlen PP, Angeles AP, Kreis RW, et al. Scar assessment tools: implications for current research. Plast Reconstr Surg 2002;109:1108-22.

2. Sullivan T, Smith J, Kermode J, et al. Rating the burn scar. J Burn Care Rehabil 1990;11:256-60.

3. Beausang E, Floyd H, Dunn KW, et al. A new quantitative scale for clinical scar assessment. Plast Reconstr Surg 1998; 102:1954-61.

4. Draaijers LJ, Tempelman FR, Botman YA, et al. The patient and observer scar assessment scale: a reliable and feasible tool for scar evaluation. Plast Reconstr Surg 2004;113:1960-5.

5. Fearmonti RM, Bond JE, Erdmann D, et al. The modified Patient and Observer Scar Assessment Scale: a novel approach to defining pathologic and nonpathologic scarring. Plast Reconstr Surg 2011;127:242-7.

6. Fearmonti R, Bond J, Erdmann D, et al. A review of scar scales and scar measuring devices. Eplasty 2010;10:e43.

7. Quinn JV, Drzewiecki AE, Stiell IG, et al. Appearance scales to measure cosmetic outcomes of healed lacerations. Am J Emerg Med 1995;13:229-31.

8. Truong PT, Lee JC, Soer B, et al. Reliability and validity testing of the Patient and Observer Scar Assessment Scale in evaluating linear scars after breast cancer surgery. Plast Reconstr Surg 2007;119:487-94.

9. Singer AJ, Arora B, Dagum A, et al. Development and validation of a novel scar evaluation scale. Plast Reconstr Surg 2007; 120:1892-7.

10. Nicholas RS, Falvey H, Lemonas P, et al. Patient-related keloid scar assessment and outcome measures. Plast Reconstr Surg 2012;129:648-56. 